\title{
MHD mixed convection boundary layer flow toward a stagnation point on a vertical surface with induced magnetic field.
}

\begin{abstract}
In this paper, the steady magnetohydrodynamic (MHD) mixed convection stagnation point flow of an incompressible, viscous, and electrically conducting fluid over a vertical flat plate is investigated. The effect of induced magnetic field is taken into account. Numerical results are obtained using an implicit finite-difference scheme. Both assisting and opposing flows are considered. The results for skin friction, heat transfer, and induced magnetic field coefficients are obtained and discussed for various parameters. The velocity, temperature, and induced magnetic field profiles are also presented. For the case of the opposing flow, it is found that dual solutions exist for a certain range of the buoyancy parameter. Dual solutions are also obtained for the assisting flow.
\end{abstract}

Keyword: Boundary layer; Dual solutions; Induced magnetic field; MHD; Mixed convection. 